\title{
Environmental Risk Assessment and Preventive Conservation Strategy for the Porch of the Glory, Santiago of Compostela Cathedral
}

\author{
Francesca Becherini ${ }^{1}$, Adriana Bernardi ${ }^{1}$, Arianna Vivarelli ${ }^{1}$, Luc Pockelé ${ }^{2}$, Sandro De Grandi ${ }^{2}$, Alessandra \\ Gandini $^{3}$, Oihana García ${ }^{3}$, Mikel Zubiaga ${ }^{3}$, Juan Carlos Espada Suárez ${ }^{3}$ and Bernardino Sperandio ${ }^{4}$ \\ 1. Institute of Atmospheric Sciences and Climate, National Research Council, Padova 35127, Italy \\ 2. Research and Environmental Devicessrl, Padova 35129, Italy \\ 3. TECNALIA Research \& Innovation, Derio 4816, Spain \\ 4. Cooperativa Beni Culturali, Perugia, Spoleto 06049, Italy
}

Received: February 27, 2013 / Accepted: April 28, 2013 / Published: May 20, 2013.

\begin{abstract}
In the framework of the Santiago of Compostela Cathedral program, a multidisciplinary investigation of the porch of the glory was carried out between 2009 and 2011 to identify the main environmental risks and to develop a preventive conservation planto be integrated in the general management strategy of the Cathedral. The study included historic and archivist research, structural studies, mineralogical analyses, biological sampling, cleaning tests and microclimatic monitoring. The main weathering factors and the related damage processes were identified. Results have shown that the main responsible for the observed damage was the infiltration of rainwater through the roof, due to cracks in the structure of the Cathedral. Other environmental factors having a remarkable impact on the state of conservation of the polychrome and its substrate were the solar radiation, the thermo-hygrometric cycles, the particle deposition and the biological growth. Solutions were suggested to improve the environmental conditions, thus reducing further damage.
\end{abstract}

Key words: Risk assessment, preventive conservation strategy, microclimatic monitoring, cultural heritage conservation.

\section{Introduction}

The Santiago of Compostela Cathedral is one of the main destinations of pilgrimage since the Middle Ages. It was declared heritage of cultural interest in 1896 and the old city of Santiago the Compostela, in which the Cathedral is situated, the UNESCO world heritage site in 1885. The porch of the glory constitutes the closure of the cathedral west facade and it was carved by Master Matthew between 1168 and 1188, with its 200 sculpted figures representing the Last Judgment, it is a masterpiece of Romanesque sculpture and it represents the most distinctive and recognized beauty

Corresponding author: Francesca Becherini, Ph.D., researcher, research fields: environmental physics, climatology and microclimatology, conservation of cultural heritage, indoor comfort and air quality. E-mail: f.becherini@isac.cnr.it. of the Cathedral.

The "Santiago of Compostela Cathedral Program" (2006-2012), financed by the Foundation Pedro Barrié de la Maza, consisted in a multidisciplinary study of the porch of the glory for the assessment of the main environmental factors affecting the state of conservation of the materials and the development of a preventive conservation strategy and restoration plan. In particular, it contemplated the following work phases for the porch of the glory to be executed between 2009-2011: preliminary study, monitoring and diagnosis, risk assessment, preventive conservation and restoration plan.

\section{Materials and Methods}

The program started with a preliminary research in 
the archive documents about the construction phase, employed techniques, previous studies on the materials, evolution of their state of conservation and past restoration work.

The monitoring and diagnostic phase was focused on the characterization of the materials and their state of conservation. In particular, the following analyses were performed by Cooperativa Beni Culturali: static monitoring to investigate the main structural problems, ultrasonic tomography of the stone elements and mechanical laboratory tests of quarry specimens; infrared thermographic analysis of the vaulted coverage; video-endoscopic studies of the stone elements, walls and floors; georadar and magnetometric investigations on the statues; mapping of the surface biological attack, dust deposits, sub-efflorescences and efflorescences; microscopic, mineralogical and petrographic studies, and other analysis (SEM-EDXS, XRD, FT-IR, GCMS) for the characterization of the materials, microorganisms, dust composition and soluble salts.

Then, as the polychromies and stones of the porch are exposed to complex environmental dynamics that affect their state of conservation, a microclimatic analysis was performed in order to investigate the on-going processes between the atmosphere and the surfaces [1] and thus contributing to the assessment of a preventive conservation plan [2, 3]. The monitoring consisted in the measurement of the thermo-hygrometric conditions of the atmosphere at different distances from the surface, including surface temperature, following a specific methodology described elsewhere [2].

All the results of the research phase were adapted into site applicable measures, by developing an environmental risk analysis and evaluation assessment [4] that permitted establishing future management and intervention actions. Inaddition, they were the basis of the future preventive conservation strategy and restoration plan of the porch.

\section{Results and Discussion}

The structural study put in evidence that the porch suffered of serious structural problems, mainly due to the instability of the south tower of the facade affected by inclination movements and land subsidence [5]. This effect inside the porch was visible in superficial lesions and deep fissures, from which rainwater infiltrated. Other cracks and lesions were due to internal defects which occurred in the plant construction consequently to the installation and the settling of the stone blocks.

The porch was realized with three types of stones: marble, coarse-grained granite and fine-medium grained granite, with different mineralogical composition. Mortars and plasters are nomore original materials but they belong to different ages and interventions. The stone materials and the plasters suffered of a quite large number of damage processes such as discoloration and stains caused by the remains of organisms, dust and dirt associated with percolation; efflorescences of nitrates and chlorides coming from percolations of water into the structure and deposition of marine aerosol; seritic alteration, arenization, pulverization, fractures and loss of fragments of material (Fig. 1) [6].

The visible paint layer belongs to different epochs, as result of the restoration interventions occurred over time, hence it differs in quality and technology. The most recent one is of the 17th century and it is based on oil technique. The main damage was the detachment and falling of painting, mainly due to the crystallization of soluble salts and to the weathering of the preparatory layer. White and dark discoloration

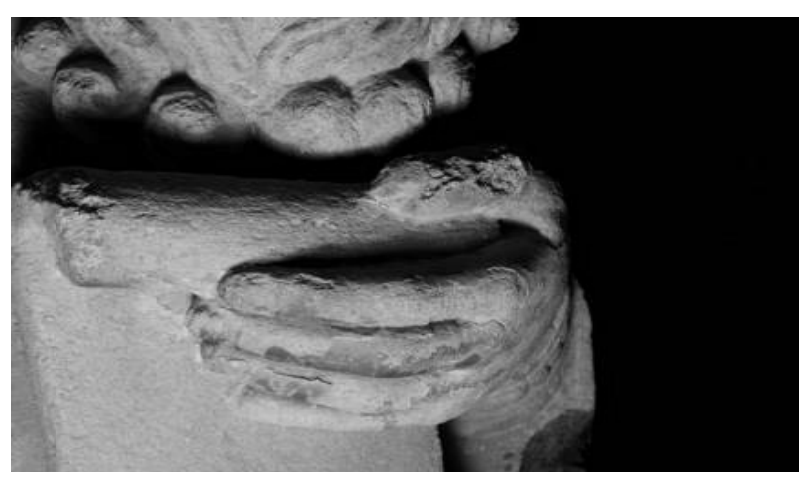

Fig. 1 Deterioration processes affecting the porch of the glory: arenization due to seritic alteration of the granite. 
due to deposition of gypsum and oxalates were also observed, as well as sub-efflorescences and efflorescences of several saline species, such as sulfates, nitrates and chlorides (Fig. 2) [6].

The video-endoscopic investigations behind the statues of the tympanum revealed the presence of vegetal substrates of soil and zone of accumulation of water drops. The biological growth covered also the surface of stone and plaster materials with a degree of contamination generally medium-low, only exceptionally high, consisting of green and black patinas, dark active colonies, presence of lichens and algae in the layer of dust. The microorganisms responsible for this degradation were mainly fungi, yeasts and algae [6].

Most of the surfaces of the porch were subject to a considerable deposition of dust. Three types of powder were identified, with different size and degree of coherence: thick layer $(>2 \mathrm{~mm})$ consistent and hardened; layer less thick and adherent $(>1 \mathrm{~mm}<2$ $\mathrm{mm})$; thin and incoherent layer $(<1 \mathrm{~mm})$. The dust layers were subjected to humidification and dehumidification cycles, due to the presence of moisture within the wall and on the surface especially in the area close to the rainwater infiltration; the powder formed a compact layer well adherent to the surface and the presence of water enhanced the development of microorganisms.

Solar radiation has a notable influence on the thermal behavior of the tympanum. In fact, the porch is oriented toward west and, in the second part of the day, the sun hits the stone surface of the tympanum through the windows of the main facade; the portion of the surface irradiated and the intensity of the radiation depend on the hour, on the season and on the climatology. As for the conservation of the polychrome the thermal variations are much more dangerous than high thermal levels, a statistical analysis of the daily thermal variations was performed in the north and south sides of the tympanum. The risk of thermal stress was more remarkable in spring and

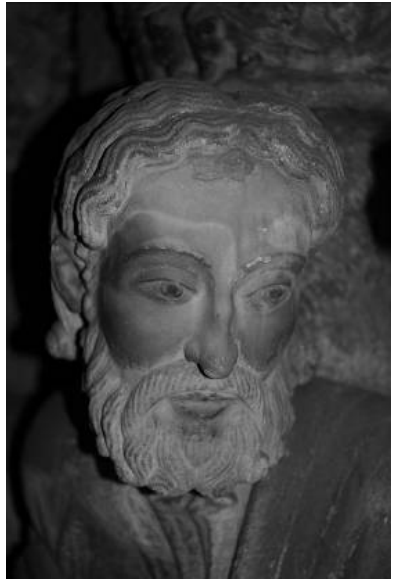

(a)

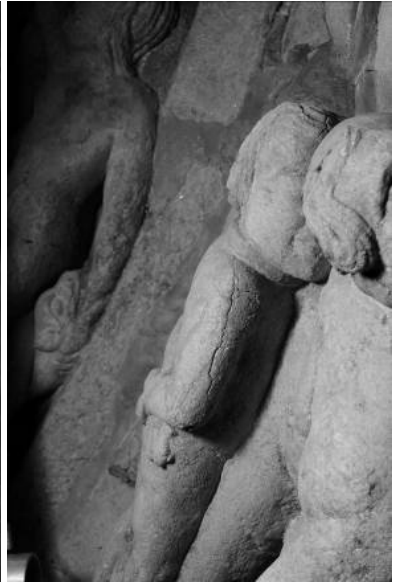

(b)
Fig. 2 Deterioration processes affecting the porch of the glory: (a) efflorescences on the edges of polychromy; (b) microfractures .

summer for the high relief of the south side, as the solar maps made by Cooperativa Beni Culturali documented [5]. In this point the daily variations were more frequent in the range $1-2{ }^{\circ} \mathrm{C}$ and they were higher than $2{ }^{\circ} \mathrm{C}$ for a third of the total time (1 year), with a maximum of $5{ }^{\circ} \mathrm{C}$. In the moments of direct solar irradiation in summer (around 20:00-20:30) the surface temperature of the south point sometimes increases of more than $1^{\circ} \mathrm{C}$ in about $1 \mathrm{~h}$ [7].

The hygrometric values in the porch were generally quite high, with an annual average of $73 \%$ and maxima frequently reached in autumn and winter. For $55 \%$ of the total time (1 year) the relative humidity was higher than $65 \%$, that is the reference value for the risk of biodeterioration [8]. The annual distribution of the hygrometric cycles, that may cause the dissolution/crystallization of the soluble salts, indicates that the cycles of $75 \%$ RH occurred for $6 \%$ of the total time (about 140 cycles in 1 year, with a rate of sampling of $3 \mathrm{~h}$ ). $75 \%$ is about the equilibrium $\mathrm{RH}$ value of $\mathrm{NaCl}$, responsible for the seritic alteration of the granite, a damage process mainly evident in the thin elements of the figures (fingers, curls) (Fig. 1) [9].

The study of the thermo-hygrometric conditions close to the surface of the tympanum allowed to evaluate the deposition of particles on the surface, in 
particular the impact of two phoretic processes [10, 11]. Results shown that for most of the year the termophoresis was inhibited, because for the most of the time surface temperature was higher than air temperature. Contrariwise, the diffusiophoresis was slightly favored ( $60 \%$ of total time) and it was more probable in winter and early spring. Moreover, during the whole year of monitoring, the study of the vertical thermal profile showed that the upper part of the porch was characterized by instability, thus favoring vertical motions of air masses and so transport of suspended particles [7]. The removal of the scaffolding, necessary to make the higher part of the porch accessible to the scientific investigations, would enhance stability favored by the entrance of external air from the front doors, generally characterized by lower temperature. Nevertheless, the absence of thermal equilibrium between air and surfaces would always favor vertical air flow along the walls where the geometry of the high-reliefs will cause local turbulence, so inertial deposition of the fine particles (1-2 $\mu \mathrm{m})$ [11].

\section{Conclusions}

The multidisciplinary study allowed an integrated assessment of the main environmental risks for the conservation of the porch of the glory and their prioritization according to their potential of damage [4]. The main environmental phenomena having a remarkable impact on the state of conservation of the polychrome and its substrate were singled out:

- rainwater infiltration through the roof, due to failures in the structure of the cathedral, that mobilized the soluble salts and favored the capture by the surfaces of the suspended particles;

- impact of the solar radiation, that enhanced thermal stress, that forced the evaporation of the filtrated water and the crystallization of the soluble salts, with phenomena of sub-efflorescences and efflorescences, crackling, detachment and loss of material (especially of the paint layer);
- particle deposition, responsible for blackening and chemical interaction with the substrate, in particular in presence of water (infiltration or condensation);

- biodeterioration, favored by the presence of water and the high values of relative humidity.

Once the environmental factors responsible for the most important damage processes at the porch had been identified and prioritized, several actions listed hereunder were suggested in order to stop these processes or at least reduce the circumstances favoring them.

First of all the technical direction of the program during the development of the project produced a plan for the repair of the cover between the two towers and the gallery above the porch, in order to stop the rainwater infiltration. The work was made between December 2010 and January 2011 and this intervention improved quickly the situation.

Then, to stabilize the microclimate, it was suggested to reduce the exchange between the internal and external atmospheres, for example repairing the windows and doors, especially the south one that presented deep fissures. Moreover, the use of the doors (especially the north one) should be controlled, reducing it on the occasion of special events, in line with the management of the access to the Cathedral. A better management of the opening/closing of the doors will surely contribute to reduce the turbulence, so the inertial deposition, caused by the atmospheric instability in the upper part of the porch. The short term analysis performed during the occasional removal of the scaffolding showed more stability but higher ventilation, two opposite conditions for the risk of the inertial deposition. Anyhow, deeper analysis by mean of a fluid dynamic model would be useful to draw final conclusions on the management of the openings.

The impact of IR and UV radiations on the surface of the tympanum, the most dangerous for conservation purposes [12], should be stopped by the installation of 
specific filters on the windows of the facade.

Last, but not least, a better management of the pilgrims' access to the Cathedral is advisable, as they are one of the main vectors of pollutants and water (especially in rainy days).

The suggested interventions, in order to be effective and sustainable, need to be integrated in the general management strategy for the conservation of the whole Cathedral.

\section{Acknowledgments}

The authors would like to give thanks to the Archbishop of Santiago, the Cathedral Chapter, the Barrié Foundation, the Regional Ministry of Culture of the Xunta de Galicia and the Technical Direction of the Spanish Institute of Cultural Heritage of the Ministry of Culture, Regional Ministry of Culture of the Xunta de Galicia, Artelan.

\section{References}

[1] D. Camuffo, Microclimate for Cultural Heritage, Elsevier, Amsterdam, 1998.

[2] A. Bernardi, Microclimate Inside Cultural Heritage Buildings, Il Prato, Padova, 2009.

[3] D. Camuffo, P. Brimblecombe, H.J. Busse, R. Van Grieken, G. Sturaro, A. Valentino, et al., Environmental risk to cultural heritage related to unsound of technology and mass tourism at the Correr Museum, Venice, The Science of the Total Environment 236 (1999) 135-152.

[4] A. Bernardi, F. Becherini, L. Pockelé, S. De Grandi, A. Gandini, O. García, et al., Monitoring of the
Environmental and Thermo-Hygrometric Parameters in the Major Chapel and the Porch of the Glory of the Santiago of Compostela Cathedral, Strategy of Control and Management of the Environmental Risks, Scientific Report for Pedro Barrié de la Maza Foundation, Pontevedra, 2011. (in Spanish)

[5] B. Sperandio, Static Monitoring of the Santiag of Compostela Cathedral and diagnostic Analyses for the Restoration Work of the Porch of the Glory, Scientific report for Pedro Barrié de la Maza Foundation, 2009. (in Italian)

[6] B. Sperandio, Investigations Performed Within the First and Second Phases of the Restoration Work of the Porch of the Glory, Scientific Report for Pedro Barrié de la Maza Foundation, 2011. (in Spanish)

[7] A. Bernardi, F. Becherini, L. Pockelé, S. De Grandi, A. Gandini, O. García, et al., Final Report of the First Phase of Investigation for the Elaboration of the Preventive Conservation Plan, Monitoring of the Microclimate in the Porch of the Glory and in the Major Chapel of the Santiago of Compostela Cathedral, Scientific report for Pedro Barrié de la Maza Foundation, 2011. (in Spanish)

[8] S. Michalski, Guidelines for humidity and temperature for Canadian archives, in: CCI Technical Bulletin 23, Canadian Conservation Institute, Ottawa, 2000.

[9] A. Goudie, H. Viles, Salt Weathering Hazards, John Wiley \& Sons, Chichester, 1988.

[10] S.K. Friedlander, Smoke, Dust And Haze, Wiley, New York, 1977.

[11] J.H. Seinfeld, Atmospheric Chemistry and Physics of Air Pollution; Wiley, New York, 1986.

[12] D. Camuffo, A. Bernardi, E. Pagan, F. Becherini, The impact of heating, lighiting and people in re-using historical buildings: A case study, Journal of Cultural Heritage 5 (2004) 409-416. 